\title{
NUMERICAL STUDIES BASED ON THE HIGHER ORDER HEAT CONDUCTION THEORY
}

\author{
M. Repka*, J. Sladek ${ }^{* *}$, V. Sladek ${ }^{* * *}$
}

\begin{abstract}
The higher order heat conduction equation is considered to study a one dimensional problem. The related boundary value problem is solved numerically via finite element method and compared with analytical solution. The influence of the characteristic time parameter from generalized heat conduction equation is investigated in numerical examples.
\end{abstract}

Keywords: Higher order heat conduction, gradient of temperature theory, finite element method.

\section{Introduction}

Due to the recent advances in manufacturing and engineering the sophisticated small scale structures are more often used in modern devices. Higher order gradient effects can play an important role in the diffusion of atoms and heat in nano-sized structures (Gad-el-Hak, 2004) or in the presence of strong gradients as in thermal shocks. To include these effects the, the classical partial differential equation needs to be enhanced by higher order spatial gradients or higher order time derivatives. Similar extensions hold for the heat equation, as advocated by Cattaneo (1958) who introduced the heat flux rate in a double temperature mixture type theory. In work of (Forest and Amestoy, 2008) the micromorphic approach has been applied to derive two types of generalized heat equations. Later (Forest and Aifantis, 2010) compared the results obtained from double temperature theory and micromorphic theory which gives a rise to associated concepts of "hypertemperature" and hyperentropy". Both concepts are described by higher-order differential equations and have been applied successfully for short-pulse laser observations in metals and treatments of cancer affected skin (Forest and Aifantis, 2010).

It is the aim of this paper study a simplified generalized heat conduction equations based on the Cattaneo's form and investigate the influence of the characteristic time parameter that appears in higher order heat conduction equation. To this end the analytical solution of the related boundary value problem has been derived in order to verify numerical solution obtained by using the finite element method.

\section{Statement of the problem}

The Cattaneo's first generalization of the heat equations has the form (Forest and Aifantis, 2010):

$$
c \rho \dot{\theta}(\mathbf{x}, \tau)=k_{i j} \theta_{, i j}(\mathbf{x}, \tau)-\theta_{0} A_{i j} \dot{\theta}_{, i j}(\mathbf{x}, \tau)
$$

\footnotetext{
Ing. Miroslav Repka, PhD.:Institute of Construction and Architecture, Slovak Academy of Sciences, Dúbravská cesta 9; 84503 Bratislava; SK, miroslav.repka@savba.sk

** Prof. Jan Sladek,DrSc.:Institute of Construction and Architecture, Slovak Academy of Sciences, Dúbravská cesta 9; 84503 Bratislava; SK, jan.sladek@savba.sk

*** Prof. Vladimir Sladek,DrSc.:Institute of Construction and Architecture, Slovak Academy of Sciences, Dúbravská cesta 9; 84503 Bratislava; SK, vladimir.sladek@savba.sk
} 
Where $k_{i j}, \rho$ and $c$ are the thermal conductivity tensor, mass density and specific heat. The additional material parameter $A_{i j}$ represents a higher order effect in an anisotropic heat-conducting solid. The reference temperature is denoted as $\theta_{0}$. The derivation of above equation is based on a modification of Fourier's heat conduction law and it deviates from classical heat conduction equation by an additional contribution which is proportional to the Laplacian of the temperature rate of change in an isotropic case. By reducing the number of new material parameters to one, $A_{i j}=\frac{\tau_{0}}{\theta_{0}} k_{i j}$, the equation (1) can be rewritten:

$$
c \rho \dot{\theta}(\mathbf{x}, \tau)=k_{i j}\left(\theta_{, i j}(\mathbf{x}, \tau)-\tau_{0} \dot{\theta}_{, i j}(\mathbf{x}, \tau)\right)
$$

The characteristic time which appears in the additional term on the right side of equation (2) is denoted as $\tau_{0}$. The weak form of the governing equation (2) can be derived from the principle of virtual work:

$$
\int_{V}\left(\lambda_{i} \delta \theta_{, i}-\tau_{0} \dot{\lambda}_{i} \delta \theta_{, i}-\rho c \dot{\theta} \delta \theta\right) d V=\int_{\Gamma_{\lambda}}\left(\bar{\Lambda}-\tau_{0} \dot{\bar{\Lambda}}\right) \delta \theta d \Gamma
$$

where the heat flux vector is denoted as $\lambda_{i}=-k_{i j} \theta_{, j}$ and scalar product $\Lambda:=n_{i} \lambda_{i}$

The equation (3) has been implemented into the commercial finite element software Comsol via the weak form module where the user written partial differential equations can be implemented.

\section{Analytical and numerical solution}

Let us consider a one dimensional problem (1-D) problem of wall with initial condition $\theta(x, 0)=0$ and prescribed temperatures on both surfaces $\theta(0, \tau)=0$ at $x=0$ and $\theta(L, \tau)=\mathrm{Te}^{-\kappa \tau} H(\tau-0)$ at $x=\mathrm{L}$ where $\mathrm{L}$ is the thickness of the wall and $H(\tau-0)$ is the Haeviside step function. For isotropic material parameters the higher order heat conduction equation (1) is simplified:

$$
\dot{\theta}(x, \tau)-a^{2} \theta^{\prime \prime}(x, \tau)+g^{2} \dot{\theta}^{\prime \prime}(x, \tau)=0
$$

Where $a^{2}=k / \beta, g^{2}=k \tau_{0} / \beta, \beta=\rho c$,

The exact analytical solution for governing equation (4) with the prescribed boundary conditions above is following:

$$
\theta(x, \tau)=\frac{T x}{L} e^{-\kappa \tau}+\sum_{n=1}^{\infty} \frac{(-1)^{n}}{\alpha_{n}-\kappa \lambda_{n}} \frac{2 T}{n \pi}\left(\left(\alpha_{n}+\kappa \beta_{n}\right) e^{-\gamma_{n} \tau}-\kappa e^{-\kappa \tau}\right) \sin \left(n \pi \frac{x}{L}\right)
$$

Where $\alpha_{n}:=\left(n \pi \frac{a}{L}\right)^{2}, \beta_{n}:=\left(n \pi \frac{g}{L}\right)^{2}, \lambda_{n}:=1-\beta_{n}, \gamma_{n}:=\frac{\alpha_{n}}{\lambda_{n}}$ 

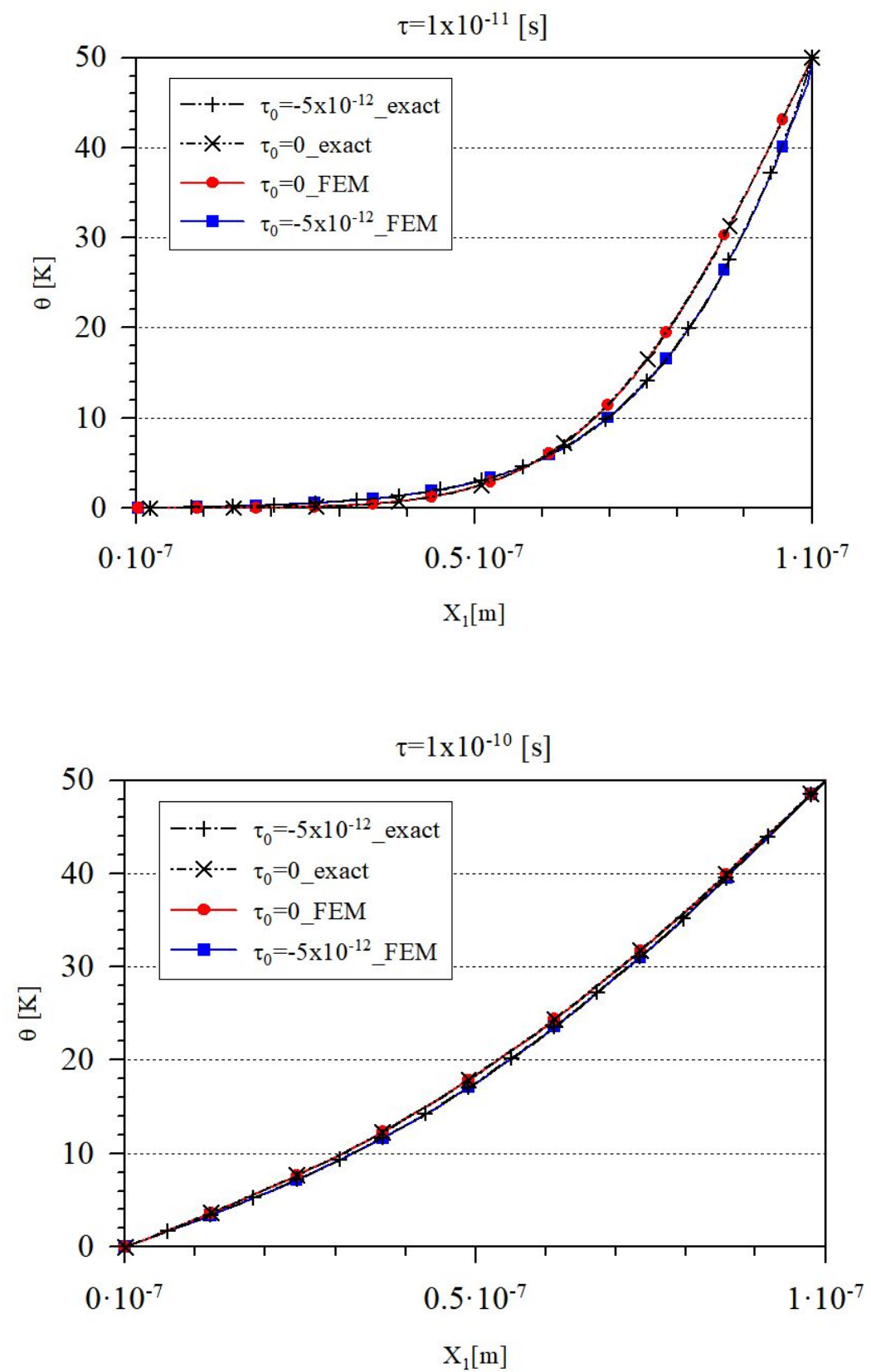

Fig. 1: Distribution of the temperature along the $x$ coordinate for various characteristic times in gradient heat conduction theory for different times, $1 \times 10^{-11}[\mathrm{~s}]$ (upper figure) and for $1 \times 10^{-10}[\mathrm{~s}]$ (lower figure).

The FEM computed variations of the temperature along $x_{1}$-coordinate axis at $\tau=10^{-11} s$ and at $\tau=10^{-10} s$ for two characteristic times, namely, $\tau_{0}=0$ and $\tau_{0}=-5 \times 10^{-12} \mathrm{~s}$, are as shown in Fig. 1 for $\kappa=0$. If $\tau_{0}=0$, the solution corresponds to classical heat conduction theory. Also shown in the figure are the exact analytical solutions obtained from (5) for these two characteristic times. It can be seen that there is very good agreement indeed between the results obtained from the FEM analysis and the analytical solutions for both the classical and gradient theory of heat conduction. It is worth noting that the temperature obtained by gradient theory $\left(\tau_{0} \neq 0\right)$ at a given position is slightly lower than that obtained from the classical theory. The influence of the characteristic time parameter is more pronounced 
for shorter time instants after application of thermal shock with presence of stronger gradients. Fig. 1 (upper) than for larger time instants when the solution reaches steady state Fig. 1 (lower).

\section{Conclusions}

The influence of the characteristic time parameter from higher order heat conduction equation has been investigated in this paper. The finite element method has been applied to solve the related boundary value problem. The numerical results have been verified with exact analytical solution. The influence of the characteristic time parameter is more pronounced for shorter time instants with the presence of the stronger gradients.

\section{Acknowledgement}

The authors acknowledge the support by the Slovak Science and Technology Assistance Agency registered under number SK-CN-RD-18-0005, and APVV-14-0440.

\section{References}

Cattaneo, M. (1958) Sur la forme de l'equation de la chaleur eliminant le paradoxe d'une propagation instantanee, Comptes Rendus de l’ Academie des Sciences Paris 247 ,pp 431-433

Forest, S. and Amestoy, M. (2008) Hypertemperature in thermoelastic solids, Comptes Rendus Mecanique 336 pp 347-353.

Forest, S., Aifantis, E.C. (2010) Some links betwe en recent gradient thermo-elasto-plasticity theories and the thermomechanics of generalized continua, International Journal of Solids and Structures 47 pp 3367-3376.

Gad-el-Hak, M. (2004) MEMS-Introduction and Fundamentals. CRC, Taylor \& Francis, London, UK. 